Chancen und Risiken in der Unternehmensführung

\section{Nachhaltigkeitsmanagement als Querschnittsaufgabe}

\author{
Nachhaltigkeitsthemen sind in der Unternehmensführung an- \\ gekommen. In Verhandlungen im Finanzsektor spielen Nach- \\ haltigkeitsaspekte allerdings noch eine untergeordnete Rolle. \\ Welche Bedeutung haben diese Erkenntnisse für die unterneh- \\ merische Praxis in Bezug auf Strategie und Berichterstattung? \\ Von Martin Viehöver und Franz Knecht
}

$\mathrm{m}$ m vergangenen Jahr beteiligten sich 37 Großunternehmen und zwei Wirtschaftsinstitutionen, die in Nachhaltigkeitsfragen im deutschsprachigen Raum tätig sind, an einer Befragung zu dem Thema „Die Bedeutung von Nachhaltigkeitsthemen in der Unternehmensführung“. Abgefragt wurde auch die wahrgenommene Relevanz dieser Themen bei der Interaktion mit dem Finanzsektor. Dabei stellte sich heraus, dass Nachhaltigkeitsthemen nicht ausreichend berücksichtigt werden, es aber zu erwarten ist, dass sich dies in Zukunft ändern wird. Tatsächlich zeigen neueste Studien, dass Investoren und Analysten zunehmend

Nachhaltigkeitsthemen unter dem Aspekt des Risiko-Management berücksichtigen (Jaworski 2007, Bassen 2006). Im Fachjargon werden diese als Extra-financials oder Environmental, sowie als Social and Governance Issues bezeichnet. Auch die zunehmende Akzeptanz und Bedeutung der „Principles for Responsible Investment" der Vereinten Nationen sowie die steigende Bedeutung im Merger \& Aquisitions-Geschäft, dem Kauf und Verkauf von Unternehmen, bestätigen die größer werdende Aufmerksamkeit des Finanzsektors.

Berichtet wurde hierbei vor allem der positive Einfluss „extra-finanzieller“ In- formationen auf Reputation und die Marke eines Unternehmens, wobei primär der Schutz dieser im Vordergrund steht. Dem Einfluss „extra-finanzieller“ Leistungen auf Firmenwert und Rendite wurde dagegen eine geringere Bedeutung beigemessen. Vertreter des Finanzsektors weisen allerdings auch immer wieder darauf hin, dass „extra-finanzielle“ Informationen, wie sie von Unternehmen in Nachhaltigkeits- oder Geschäftsberichten dargestellt werden, kaum einen Rückschluss darauf zulassen, inwieweit diese Faktoren die finanzielle Performance eines Unternehmens wirklich beeinflussen.

\section{Bedeutung für Unternehmen}

Das Defizit erklärt sich zum einen durch die zum Teil methodische Schwierigkeit, den Einfluss qualitativer Faktoren auf quantifizierbare Effekte zu messen. Zum anderen wird in vielen Unternehmen kaum der Versuch unternommen, diesen Einfluss zu messen. Vielfach scheint auch eine auf die Nachhaltigkeitsthemen des Unternehmens zugeschnittene, vom Vorstand getragene und konsequent umgesetzte Nachhaltigkeits- oder Corporate-Responsibility-Strategie zu fehlen, die aber die unabdingbare Grundlage dafür ist (Kirchhoff 2007).

Um den Anforderungen des Finanzsektors effizient $\mathrm{zu}$ entsprechen, bedarf es der gezielten Integration von Corporate Responsibility-Aspekten in das bestehende Managementsystem. Hierbei spielt neben der Frage nach der Verankerung auch die Einbindung von Fachabteilungen, die in Nachhaltigkeitsfragen fachlich kompetent sind, eine große Rolle. Welche Bereiche aus Sicht der befragten Unternehmen heute und künftig besonders von Nachhaltigkeits- 
themen betroffen sind beziehungsweise es sein werden, ist in Tabelle 1 dargestellt.

Derzeit sind die Geschäftsleitung sowie die Bereiche Kommunikation und Public Relations am stärksten durch Chancen und Risiken aus Nachhaltigkeitsthemen betroffen. Auch die Bereiche Planung und Strategie erhielten ähnliche Nennungen. Bei der Einschätzung zukünftiger Chancen liegen die Bereiche Kommunikation und Public Relations deutlich an der Spitze, dem folgen Geschäftsleitung sowie Planung und Strategie.

Auch hier zeigt es sich, dass der Kommunikation eine Schlüsselrolle zugewiesen wird, was sich auch aus dem zunehmenden Informationsbedarf des Finanzsektors schließen lässt. Die Vervierfachung der Betroffenheit des Merger \& Aquisitions- Bereichs ist auch ein Indiz dafür, dass „extra-finanzielle“ Faktoren in der Unternehmensbewertung eine größere Rolle spielen werden, als dies in der heutigen Praxis der Fall ist.

Kritisch sehen wir die Einschätzung der befragten Unternehmen, dass die Betroffenheit des Forschung und Entwicklungsbereichs abnehmen wird. Gerade für die Identifizierung und Nutzung von Chancen durch neue Produkte und Dienstleistungen, die mit einem Nachhaltigkeitsthema verknüpft sind, ist die Einbindung dieses Bereiches unabdingbar. Man denke etwa an die aktuelle Diskussion von Supply-Chain- und Globalisierungsaspekten, die gerade an die integrierte Forschung und Entwicklung neue Anforderungen stellen.

\section{Fazit}

Insgesamt rechnen die befragten Unternehmen damit, dass die verschiedenen Bereiche zunehmend mit Nachhaltigkeitsthemen konfrontiert sein werden. Dabei wird erwartet, dass die daraus entstehenden Chancen überwiegen werden, was sicherlich eine Herausforderung an die Querschnittsfunktion des Nachhaltigkeitsmanagements darstellt. Um diese Chancen auch wirklich nutzen zu können, muss aus unserer Sicht das Management in vielen Fällen um einiges strukturierter vorgehen, als dies derzeit der Fall ist.

Für die Erarbeitung einer auf das Unternehmen zugeschnittenen Nachhaltigkeits- und Corporate-Responsibility Strategie sollten möglichst alle von Nachhaltigkeitsthemen betroffenen Bereiche eingebunden werden, um einerseits die konsequente Umsetzung sicherzustellen und andererseits Fortbildungs- und Beratungsbedarf zu identifizieren. Insbesondere die Einbeziehung der mittleren Führungsebene ist oft entscheidend, wenn es um die erfolgreiche Umsetzung von Corporate-Responsibility-Strategien geht, da diese in der internen Berichterstattung eine Schlüsselfunktion einnehmen, was wiederum entscheidend für die Qualität der externen Berichterstattung ist. Um letztlich das Vertrauen von Analysten, Kapitalgebern und Investoren zu gewinnen, empfiehlt es sich, eine offene und transparente Firmenkultur zu pflegen, die in der Berichterstattung Probleme offen anspricht und Lösungsstrategien darstellt.

\section{Literatur}

Bassen, A. / Hölz, H.-M. / Schlange, J. / Meyer, K. / Zamostny, A.: The Influence of Corporate Responsibility on the Cost of Capital. Hamburg 2006

Jaworski, W.: Use of Extra-Financial Information by Research Analysts and Investment Managers. Maastricht 2007.

Kirchhoff, K.-R. / Balzer, A. / Gazdar, K. / Grewe, W. / Günther, E. / Habisch, A. / Hecht, J.: Das Good Company Ranking. Hamburg 2007.

Viehöver, M. / Hilf, J. / Knecht, F. / Reich, S.: Nachhaltigkeit und Unternehmensführung. Frankfurt am Main, Basel, Köln 2006. Verfügbar unter: http://www.urscorp.de/index.php? getfile=2006-11-09_nachhaltigkeit_und_unternehmensfuehrung_2006.pdf

\section{AUTOREN + KONTAKT}

Martin G. Viehöver leitet die Gruppe Unternehmensverantwortung und Nachhaltigkeit von URS Deutschland $\mathrm{GmbH}$.

URS Deutschland GmbH, Tel.: 06103/9389-36, E-Mail:martin_viehoever@urscorp.com

Franz Knecht ist Geschäftsführer der Beratungsfirma Connexis Strategy Consultants AG mit Sitz in Basel.

Connexis Strategy Consultants AG, Tel.: +41-61/2724203,

E-Mail: franz.knecht@connexis.ch

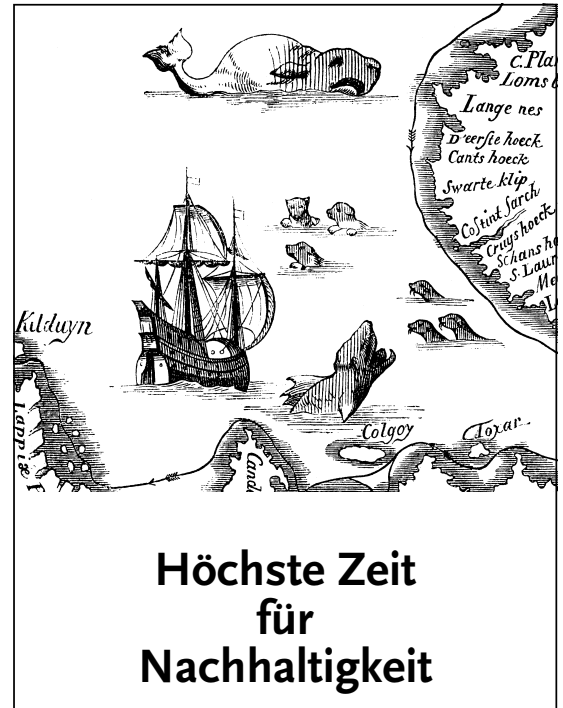

Der Klimawandel hat die Köpfe erreicht. Doch reicht es aus, ihm zu begegnen, indem Energie gespart und Erneuerbare Energien verstärkt genutzt werden? Muss nicht der Ressourceneinsatz insgesamt deutlich gesenkt werden?

factor $Y_{\text {ist das }}$

Magazin für Nachhaltiges Wirtschaften.

factorY ist den aktuellen Entwicklungen für Nachhaltigkeit in Produktion und Konsum nachgegangen.

factorY stellt Unternehmen vor, die in der gesamten Produktionskette nachhaltige Strukturen schaffen.

factorY $Y_{\text {spürt die Ansätze für }}$ Nachhaltigkeit in den aktuellen Konsumtrends auf.

factorY Probeabo bei factorY Publishing Am Varenholt I23, 44797 Bochum T 0234.9799513, F 0234.9799514 oder www.factory-magazin.de

Einzelheft: $4,60 €$ zzgl. Versand factorY im Abo: 4 Hefte für 23,50 € Inland, 33,50 € Ausland (inkl. MwSt und Versand)

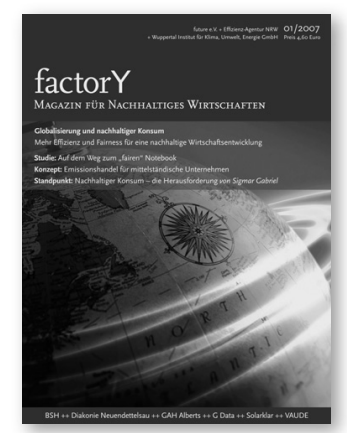


(c) 20I0 Authors; licensee IÖW and oekom verlag. This is an article distributed under the terms of the Creative Commons Attribution Non-Commercial No Derivates License (http://creativecommons.org/licenses/by-nc-nd/3.o/), which permits unrestricted use, distribution, and reproduction in any medium, provided the original work is properly cited. 